\title{
COMPASS points to low-dose rivaroxaban and aspirin for secondary prevention
}

In patients with stable atherosclerotic vascular disease, the combination of a low dose of rivaroxaban and aspirin is associated with a lower rate of cardiovascular outcomes but more major bleeding events compared with aspirin alone. This finding from the COMPASS trial was presented at the ESC Congress 2017.

The double-blind COMPASS trial involved 27,395 patients with stable atherosclerotic vascular disease (90.6\% with a history of coronary artery disease and $27.3 \%$ with a history of peripheral artery disease). Participants were randomly assigned to receive rivaroxaban $(2.5 \mathrm{mg}$ twice daily) plus aspirin (100 mg once daily), rivaroxaban (5 $\mathrm{mg}$ twice daily), or aspirin ( $100 \mathrm{mg}$ once daily). Of note, both doses of rivaroxaban were lower than the $10 \mathrm{mg}$ dose approved for stroke prevention in patients with atrial fibrillation. The independent data and safety monitoring board recommended early termination of this part of the trial (mean follow-up 23 months) because of superiority of the rivaroxaban plus aspirin group.

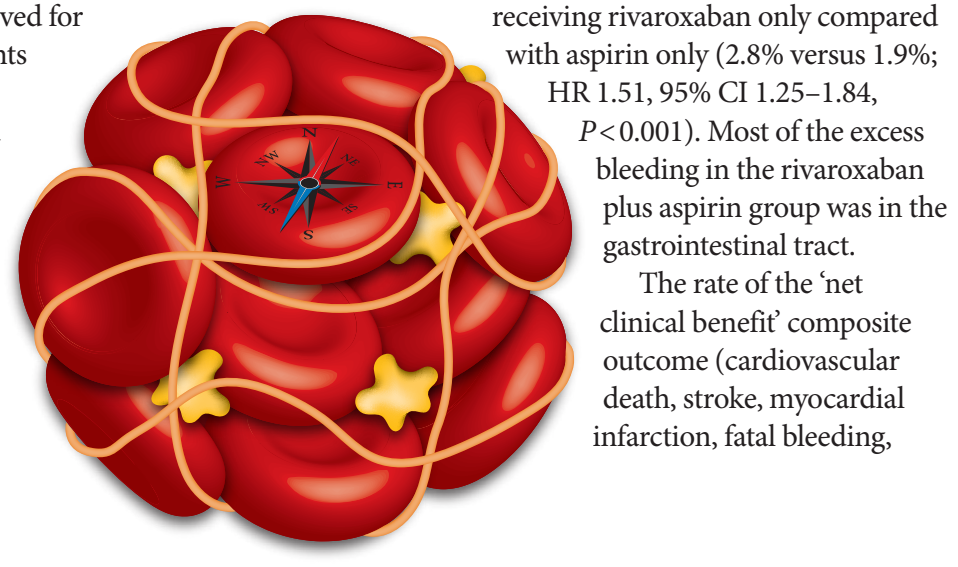
with aspirin only ( $2.8 \%$ versus $1.9 \%$; bleeding in the rivaroxaban plus aspirin group was in the astrointestinal tract. clinical benefit' composite outcome (cardiovascular death, stroke, myocardial
The rate of the primary outcome (a composite of cardiovascular death, stroke, or myocardial infarction) was significantly lower in the rivaroxaban plus aspirin group than in the aspirin-only group (4.1\% versus $5.4 \%$; HR 0.76 , 95\% CI $0.66-0.86, P<0.001$ ), but was not significantly different with rivaroxaban only compared with aspirin only (4.9\% versus 5.4\%; HR 0.90, $95 \%$ CI 0.79-1.03, $P=0.12$ ). All-cause death occurred in $3.4 \%, 4.0 \%$, and $4.1 \%$ of patients in the rivaroxaban plus aspirin, rivaroxaban-only, and aspirin-only groups, respectively.

The rate of major bleeding was higher in patients receiving rivaroxaban and aspirin than in patients receiving aspirin only (3.1\% versus 1.9\%; HR 1.70, 95\% CI 1.40-2.05, $P<0.001$ ), and higher in patients receiving rivaroxaban only compared
The COMPASS

investigators

... hit the

sweet spot

with the dose

of rivaroxaban

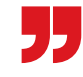

or symptomatic bleeding into a critical organ) was significantly lower with rivaroxaban plus aspirin than with aspirin alone (4.7\% versus $5.9 \%$; HR 0.80, 95\% CI 0.70-0.91, $P<0.001$ ), but was not significantly different with rivaroxaban only compared with aspirin only (5.5\% versus $5.9 \%$; HR 0.94, 95\% CI 0.84-1.07, $P=0.36$ ).

"The COMPASS investigators ... hit the sweet spot with the dose of rivaroxaban, thereby maximizing the efficacy of the combination while minimizing the bleeding risk," comments Jack Hirsh (McMaster University, Ontario, Canada). “The findings are likely to change clinical practice, because the combination not only increased net clinical benefit, but also reduced total mortality," he adds. John Eikelboom, lead author on the trial report, anticipates that the results will "lead to widespread regulatory approval for long-term secondary prevention in patients with coronary or peripheral artery disease". Nevertheless, "bleeding is always an issue ... We are currently testing whether pantoprazole can reduce the risk of gastrointestinal bleeding in patients receiving antithrombotic therapy for secondary prevention."

Gregory B. Lim

ORIGINAL ARTICLE Eikelboom, J. W. et al. Rivaroxaban with or without aspirin in stable cardiovascular disease. N. Engl.J. Med. http:// dx.doi.org/10.1056/NEJMoa1709118 (2017) FURTHER READING Capodanno, D. et al. Antithrombotic therapy for secondary prevention of atherothrombotic events in cerebrovascular disease. Nat. Rev. Cardiol. 13, 609-622 (2016) 